\title{
Multi-Spatial Classifier for Blue Whale Images using Photo-Identification Method
}

\author{
Rosa I. Ramos-Arredondo ${ }^{1}$, Blanca E. Carvajal-Gámez ${ }^{2}$, Francisco J. Gallegos- \\ Funes $^{3}$, and Diane Gendron-Laniel ${ }^{4}$ \\ ${ }^{1}$ Escuela Superior de Cómputo, Instituto Politécnico Nacional (IPN), Mexico \\ ${ }^{2}$ Unidad Profesional Interdisciplinaria en Ingeniería y Tecnologías Avanzadas, IPN, Mexico \\ ${ }^{3}$ Escuela Superior de Ingeniería Mecánica y Eléctrica, IPN, Mexico \\ ${ }^{4}$ Centro Interdisciplinario de Ciencias Marinas, IPN, Mexico \\ alesija@gmail.com, becarvajal@ipn.mx, fgallegosf@ipn.mx,dgendron@ipn.mx
}

\begin{abstract}
The process of photo-identification images of the blue whale (Balaenoptera musculus), is made manually; this process classifies images blue whale through its dorsal fin characteristics. The features are extracted visually, which can generate errors at the moment of classified. In this work an image classifier blue whale is presented, which have features such as color pigmentation, background image, type of dorsal fin, among others; these common characteristics generate high statistical dependence. This statistical dependence causes the data extracted through a segmented image of the blue whale, are to be observed through a hyperplane. Using statistical techniques and the K-Nearest Neighbors classifier, the classification of three types of dorsal fin is obtained with an accuracy of $71.66 \%$, assessing the value of $\mathrm{K}=7$, taking reference catalog of species CICIMAR- IPN.
\end{abstract}

Keywords: Classifier, K-nearest-neighbors, feature extraction, multi-spatial classifier.

\section{Introduction}

The technique of photo-identification of marine mammals started in the 70's by the marine biologist, Michael Bigg. The technical manual is considered a noninvasive method, in addition to many advantages in identifying natural markings, temporary marks and scars such as: the shape of the fins, the location of it and coloring marks [1]. These features are unique between each marine mammals [2]. One of the features used to differentiate other is the shape of its dorsal fin (fin found on the back of the body of the whale) and caudal (fin located on the lower body of the whale). Specialists in marine mammals, when conducting field survey samples, need to identify the element of study in the first instance is through photographs where the sides of your body and how their fins are displayed. It is noteworthy that the blue 
whale shows its tail fin infrequently and is due to this factor that is convenient to take as the identifier element to its dorsal fin.

Photographs of the Blue Whale were mostly taken in the Gulf of California (CICIMAR-IPN) have the particularity to be taken at sea, having among the main challenges for this analysis: the seabed, natural camouflage techniques marine animals, luminosity and different angles of the image when the picture is taken. In order to classify the element study by the type of dorsal fin need to perform a preprocessing the image without altering the morphology of the same. Later, we require segmentation stage we would help to get the shape identifier.

The classifier will give us an estimate of fin type to which it belongs. The type classification is given by dorsal contour form [3]. For the study we will focus on only three types of fin (falcate, triangular and hooked). The catalog of CICIMAR-IPN has previously classified photographs by the shape of the flap and flanks.

Extracting features of the shape of the dorsal fin factors considered scaling, translation and rotation, for example; the photographs are captured at different distances (100-300 meters). In addition a large number of images from the same individual study for the selection and classification of features to identify needs.

The article is organized as follows: Section 2 shows the methodology for classification, Section 3 describes the Hu invariant moments for generating the feature vector we need classification identifier, Section 4 shows the details of the classification, and Section 5 discusses the experiments and the results obtained. Finally, Section 6 concludes this paper.

\section{Methodology}

In this paper previous stages are required to obtain samples that will engage in training the classifier and to classify samples. Divide into two phases: the first is called sample collection and the second classification of samples. The following figures illustrate each of the phases and stages that carry a flow of execution.

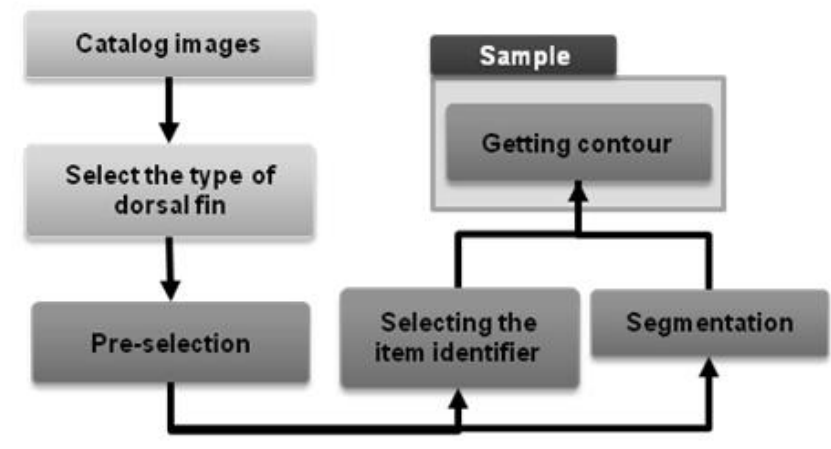

Fig. 1. First stage: sample preparation. 


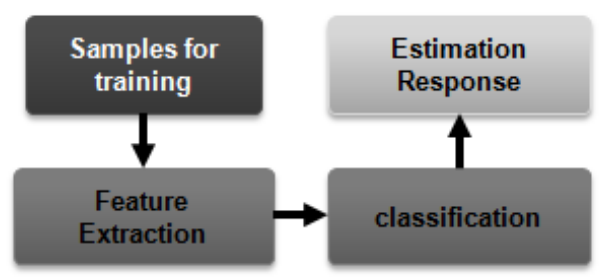

Fig. 2. Second stage: classification of samples.

\subsection{Selecting the Type of Dorsal Fin}

In the catalog of species-IPN CICIMAR we focus on the blue whale as identifying the dorsal fin is used. Photographs where the dorsal fin of the blue whale is displayed, regardless of the side will look. Another parameter in the selection list is the shape of the flap; for the study will consider three types of fins: falcate, triangular hooked. See in Figure 3, three examples of the ways of studying the dorsal fin.

Photos used within the context of article images from the call that will perform such a calculation from the pre-processing may consider the name of images. The images in the course of the study will be occupied but for the purpose of reducing the storage space on the calculation and standardize the treatment, we focus on a sample of the image where the identifier elements to classify (the dorsal fin whale blue).

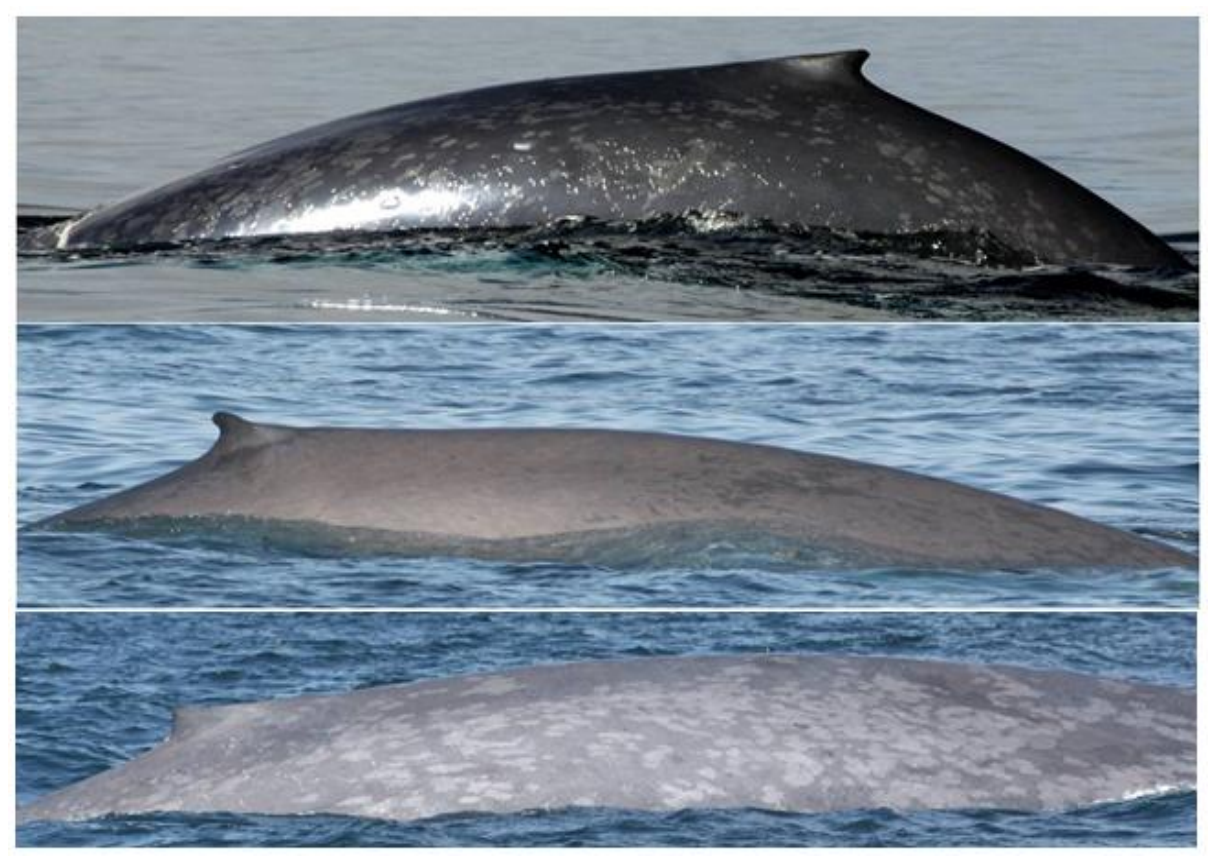

Fig. 3. Photographs of the dorsal fin. 


\subsection{Pre-Selection}

In the stage of pre-selection we find photographs that were collected either with digital or SLR cameras. The pictures that were not taken with a digital camera was to grayscale and were digitized using a scanner to have them in digital format. To standardize the photographs to use images that were formed from three layers of color (RGB - Red, Green, and Blue) were selected.

\subsection{Representation of a Photograph in a Digital Image}

The picture is represented by an image $\mathrm{f}(\mathrm{x}, \mathrm{y})$, where $\mathrm{f}$ represents the value of the light intensity at each point $(\mathrm{x}, \mathrm{y})$. .The $\mathrm{x}, \mathrm{y}$ are the spatial coordinates. $\mathrm{M}, \mathrm{N}$, are the rows and columns of the digital image [4]. This step was necessary to delimit the number of factors to consider in the photographs and the following steps are done processing.

$$
f(x, y)=\sum_{x=1}^{M} \sum_{y=1}^{N}(x, y)
$$

\subsection{Selecting the Item Identifier}

The images of the blue whale's dorsal fin where shown are not the same size when they are harvested. Taking as an advantage point to classify blue whale by the shape of its dorsal fin, select a portion of the image where the louver and we can give all the relevant information in addition to normalizing the portion size.

\subsection{Segmentation}

In [4], [5] indicates that the segmentation is one of the most important for the extraction of features and / or steps in the image recognition step. Wanting to classify blue whale through the dorsal fin, leads us to want to find the shape of the fin to differentiate between forms of fins that were raised in the study (falcate, hooked, triangular). The portion of the image has the same problems that had full but with the advantage of being more amenable to their size image. When dealing with images of blue whales has some problem like the reflection of the sun on the skin of the whale, the blue whale color with the color of the sea. The segmentation of the dorsal fin is for localizing region-based active contours. They adapt the integration of a rectangular region centered on the image so that the number of iterations is low [6]. 

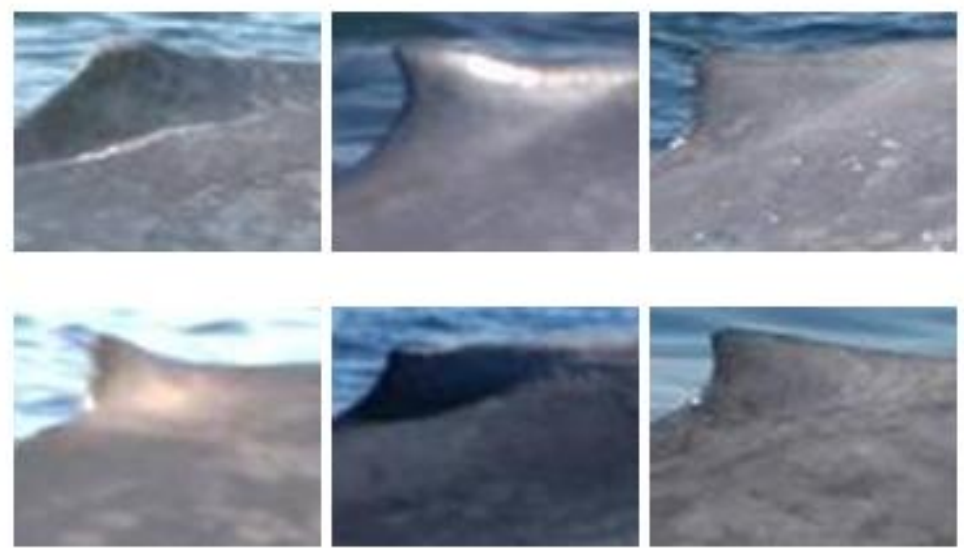

Fig. 4. Select of the dorsal fin. The images of the dorsal fin are the right flank of the blue whale. Some of the problems mentioned in the article shows.

\subsection{Getting Contour}

From the image with the segmented region have the dorsal fin separated from the image background. Obtaining the contour is to be used in stage two as a sample, wherein the extraction characteristics are performed. For this we need to use a technique that provides us the outline and the study sample can be represented in a binary image as shown in Figure 5.
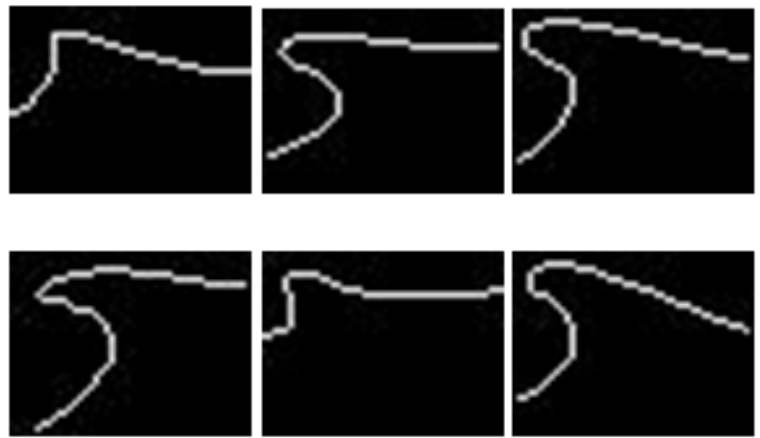

Fig. 5. Contour of dorsal fin. The contours of the dorsal fin are also called dealing samples for phase two were extracted features and the classification of the fin is made.

\section{Feature Extraction}

At this stage we focus on obtaining contour features that factor to differentiate between the types of dorsal fin shape. Three kinds of shape and take their flanks. 
There are other features that could be obtained as the wet skin texture but the blue whale with sun reflection alters the appearance, taking other factors that should be addressed from the pre-processed before being used as a feature. Another feature to consider is into account is that the photographs are not taken from the same distance and location; for this reason the seven Hu moment invariants are a good choice in feature extraction providing be invariant to changes in scale, translation and rotation $[7,8]$.

They extracted features samples of the three types of shape of the dorsal fin and seven $\mathrm{Hu}$ invariant moments, it becomes part of the feature vector to deal in the standings. In Figure 6 the Hu invariant moments ten samples of each class is plotted and where their behavior is observed.

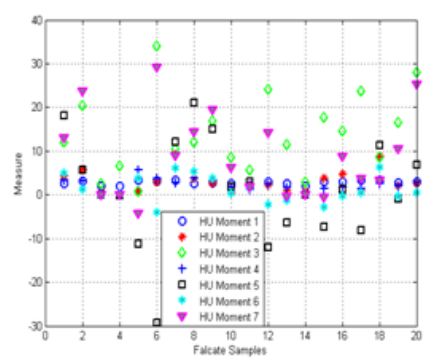

a)

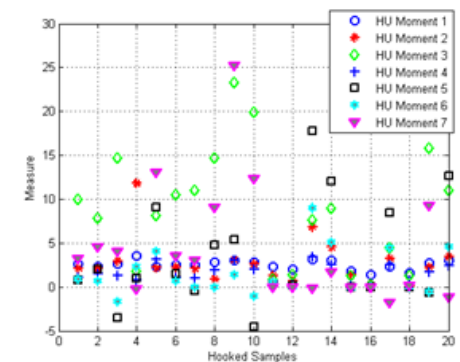

b)

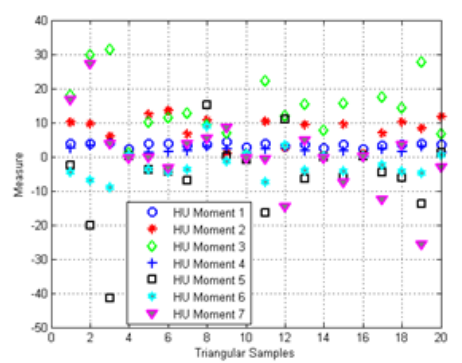

c)

Fig. 6. Is the graph of twenty samples each of the shape of the dorsal flank without differentiating which were extracted features - HU seven moments invariant time are graphed. Where, (a) Falcate class; (b) Hooked class; and (c) Triangular class.

\section{Classification}

The study supervised by taking reference a previous classification by specialists and contemplated ordering your catalog using manual techniques classification is made. The minimum distance classifier and k-Nearest Neighbors (K-NN) will look to be quick in qualifying. Although training may take a little longer by marine mammal specialists (especially if the blue whale) to select samples where the shape of the dorsal fin is representative of each class, not a negative point consider. 


\subsection{Training}

It will take at random a number of samples of each class. The classes defined are six right falcate, falcate left, right hook, left hook, right and left triangular.

\subsection{Classifiers}

For the minimum distance classifier to classify the samples are taken knowing how they belong and depending on the estimate is to determine whether the valuation is correct or incorrect [9].

In the KNN classifier takes $\mathrm{K}$ nearest neighbors of the sample to be classified. $\mathrm{K}$ is an odd integer number $K=3,5,7,9$ Starting on number of $K=5$, there may be a tie. Therefore, a tiebreaker rule is proposed. Take another neighbor and if they present another tie proceed with my order.

\section{Experiments and Results}

This section describes the experiments and their results will be discussed, taking the methodology set. The methods were tested in Matlab®.

For feature extraction are represented by the seven $\mathrm{Hu}$ moment invariants. Classes in the experiments are given as: a) Class 1-Falcate right b) Class 2 left Falcate c) Class 3 - right hook d) Class 4 - left hook e) Class 5 - Triangular right f) Class 6 Triangular left. It requires an analysis of the income generated in the experiments; calculating deal sensitivity and accuracy. [10-12]

\subsection{Minimum Distance Classifier}

Conditions for training; 10 samples of each of the classes for a total of 60 training samples were taken and to classify 20 samples of each class were taken, the results of the classification are shown in Table 1. In Table 2, one can see the experiment performance analysis.

Table 1. Experiment with the minimum distance classifier.

\begin{tabular}{lllllll}
\hline & Class 1 & Class 2 & Class 3 & Class 4 & Class 5 & Class 6 \\
\hline Estimation & 0.2 & 0.35 & 0.2 & 0.6 & 0.45 & 0.45 \\
\hline
\end{tabular}

Table 2. Evaluation of minimum distance classifier.

\begin{tabular}{ll}
\hline \multicolumn{1}{c}{ Test } & Evaluation \\
\hline Sensitivity (\%) & 37.5 \\
Precision $(\%)$ & 37.5 \\
\hline
\end{tabular}




\subsection{K-NN Classifier}

For the experiment, 10 samples of each of the classes give 60 sample are taken for the training. In the group of 20 samples of each class to class, tests are performed with the value of $\mathrm{K}=3,5,7,9$.

Table 3. Experiments with the KNN classifier with six classes (distinguishing between right and left).

\begin{tabular}{lllll}
\hline Class & $\begin{array}{l}\text { Estimation } \\
\mathrm{k}=3\end{array}$ & $\begin{array}{l}\text { Estimation } \\
\mathrm{k}=5\end{array}$ & $\begin{array}{l}\text { Estimation } \\
\mathrm{k}=7\end{array}$ & $\begin{array}{l}\text { Estimation } \\
\mathrm{k}=9\end{array}$ \\
\hline Class 1 & 0.65 & 0.5 & 0.5 & 0.5 \\
Class 2 & 0.35 & 0.35 & 0.3 & 0.35 \\
Class 3 & 0.4 & 0.3 & 0.35 & 0.35 \\
Class 4 & 0.5 & 0.55 & 0.55 & 0.55 \\
Class 5 & 0.25 & 0.3 & 0.25 & 0.25 \\
Class 6 & 0.4 & 0.45 & 0.3 & 0.45 \\
\hline
\end{tabular}

Table 4. Evaluation of K-NN classifier with six classes.

\begin{tabular}{lcccc} 
& $\begin{array}{c}\text { Evaluation } \\
\mathrm{k}=3\end{array}$ & $\begin{array}{c}\text { Evaluation } \\
\mathrm{k}=5\end{array}$ & $\begin{array}{c}\text { Evaluation } \\
\mathrm{k}=7\end{array}$ & $\begin{array}{c}\text { Evaluation } \\
\mathrm{k}=9\end{array}$ \\
\hline Sensitivity (\%) & 45.83 & 40.83 & 37.5 & 40.83 \\
Precision (\%) & 45.83 & 40.83 & 37.5 & 40.83 \\
\hline
\end{tabular}

The best result was given in which the value of $\mathrm{K}=3$ and Class 5 features in classifying low estimate.

Classes for this experiment are given by: a) Class 1 - Falcate, b) Class 2 - Hooked, c) Class 3 - Triangular. Classes include both flanks.

Table 5. Experiment with the KNN classifier regardless flanks of the dorsal fin.

\begin{tabular}{lllll}
\hline Class & $\begin{array}{l}\text { Estimation } \\
\mathrm{k}=3\end{array}$ & $\begin{array}{l}\text { Estimation } \\
\mathrm{k}=5\end{array}$ & $\begin{array}{l}\text { Estimation } \\
\mathrm{k}=7\end{array}$ & $\begin{array}{l}\text { Estimation } \\
\mathrm{k}=9\end{array}$ \\
\hline Class 1 & 0.8 & 0.8 & 0.85 & 0.75 \\
Class 2 & 0.6 & 0.45 & 0.6 & 0.65 \\
Class 3 & 0.7 & 0.65 & 0.7 & 0.55 \\
\hline
\end{tabular}

Table 6. Evaluation of K-NN classifier with three classes.

\begin{tabular}{llccc}
\hline & $\begin{array}{c}\text { Evaluation } \\
\mathrm{k}=3\end{array}$ & $\begin{array}{c}\text { Evaluation } \\
\mathrm{k}=5\end{array}$ & $\begin{array}{c}\text { Evaluation } \\
\mathrm{k}=7\end{array}$ & $\begin{array}{c}\text { Evaluation } \\
\mathrm{k}=9\end{array}$ \\
\hline Sensitivity (\%) & 70 & 63.33 & 71.66 & 65 \\
Precision (\%) & 70 & 63.33 & 71.66 & 65 \\
\hline
\end{tabular}


By grouping classes, $\mathrm{K}=7$, presented better results when classifying. Furthermore Class 2 (hooked) presented lower rate when compared classified the other two forms.

\section{Conclusions}

The K-NN classifier performs best compared to the minimum distance classifier to classify the blue whale through the dorsal fin shape, besides being of low complexity. Pre-qualifying stage must ensure are as correct because the problems they had to alter the selection of samples for training and the classifier used.

The shape of the dorsal fin shown hooked to classify a lower estimate among other way and is by a factor that alters the shape of the dorsal fin of the samples and is barnacles that stick to the flap and the body, deforming its shape. Although the classifier gives an estimation of the shape of which this may give an incorrect estimate. This factor is suggested to perform a pre-processing for the detection and / or removal of external agents that alter the shape. Classification performs better when the number of classes is reduced.

Another factor that could observe that alters the shape of the dorsal fin of the blue whale is trademarks and / or mutilation that occurs throughout life, causing a misclassification. Detection and / or correction at the stage of pre-processing are suggested.

Acknowledgments. We express our gratitude to the National Polytechnic Institute of Mexico (Instituto Politécnico Nacional) and National Council on Science and Technology of Mexico (CONACYT) for the help and support for project number 221284. Thanks for the support from the team of Diane Gendron Laniel.

\section{References}

1. Auger-Méthé, M., Whitehead, H.: The use of natural markings in studies of longfinned pilot whales (globicephala melas). Marine Mammal Science, 23(1), pp. 7793 (2007)

2. Perrin, W. F., Wursig, B., Thewissen, J. G. M. (Eds.): Encyclopedia of marine mammals. Academic Press (2009)

3. Gendron, D., \& Ugalde de la Cruz, A.: A new classification method to simplify blue whale photo-identification technique. J. CETACEAN RES. MANAGE, 12(1), pp. 79-84 (2012)

4. Gonzalez, R. C., Woods, R. E.: Digital image processing (2002)

5. Shih, F. Y.: Image processing and pattern recognition: fundamentals and techniques. John Wiley \& Sons (2010)

6. Lankton, S., Tannenbaum, A.: Localizing region-based active contours. Image Processing, IEEE Transactions on, 17(11), pp. 2029-2039 (2008)

7. Hu, M. K.: Visual pattern recognition by moment invariants. Information Theory, IRE Transactions on, 8(2), pp. 179-187 (1962) 
8. Theodoridis, S., Koutroumbas, K.: Pattern recognition. IEEE TRANSACTIONS ON NEURAL NETWORKS, 19(2), 376 (2008)

9. Lin, H., Venetsanopoulos, A.N.: A weighted minimum distance classifier for pattern recognition. In proceedings of the Canadian Conference on Electrical and Computer Engineering, vol. 2, pp.904-907, (1993)

10. Trejo-Salazar, D., Carvajal-Gámez, B.E., Gallegos-Funes, F.J.: Algoritmo de segmentación para imágenes reales para la clasificación de cetáceos. In proceedings of COMIA (2013)

11. Fathi, A., Naghsh-Nilchi, A. R.: Automatic wavelet-based retinal blood vessels segmentation and vessel diameter estimation. Biomedical Signal Processing and Control, 8(1), pp. 71-80 (2013)

12. Fraz, M. M., Barman, S. A., Remagnino, P., Hoppe, A., Basit, A., Uyyanonvara, B., Owen, C. G.: An approach to localize the retinal blood vessels using bit planes and centerline detection. Computer methods and programs in biomedicine, 108(2), pp. 600-616 (2012) 